\title{
Originales
}

\section{Valoración de la capacidad preoperatoria al ejercicio como factor predictivo de supervivencia en enfermos sometidos a trasplante pulmonar}

\author{
A. GONZÁLEZ CASTROa, B. SUBERVIOLA CAÑASa, A. QUESADA SUESCUNa, \\ M.S. HOLANDA PEÑA ${ }^{a}$ C. GONZÁLEZ FERNÁNDEZ ${ }^{a}$ Y J. LLORCA ${ }^{b}$
}

\begin{abstract}
aServicio de Medicina Intensiva. Hospital Universitario Marqués de Valdecilla. Santander. España.
${ }^{b}$ Departamento de Estadística, Medicina Preventiva y Salud Pública. Facultad de Medicina. Universidad de Cantabria. Santander. España.
\end{abstract}

Objetivo. Evaluar de manera independiente la capacidad al ejercicio como factor predictivo de supervivencia en el trasplante pulmonar.

Diseño. Estudio retrospectivo.

Ámbito. Unidad de trasplante pulmonar de la Unidad de Cuidados Intensivos (UCI) de un hospital universitario.

Pacientes. Se realizaron y analizaron 132 trasplantes pulmonares, en un total de 130 enfermos. El seguimiento de cada caso se realizó hasta fecha de 18 de junio de 2005 o fallecimiento del paciente.

Variables de interés. Se valoró la capacidad preoperatoria al ejercicio mediante la prueba de marcha de los 6 minutos (PM6M). También se valoró la supervivencia a largo plazo en relación con el valor obtenido en la PM6M. Las curvas de supervivencia se estimaron por el método de Kaplan-Meier; la comparación entre curvas se hizo por el método de Log-Rank y las hazard ratios se estimaron por regresión de Cox.

Resultados. El estudio de asociación a mortalidad de la PM6M mostró un riesgo relativo de 0,99 (intervalo de confianza del 95\%: 0,99-1,00) por cada unidad de medida métrica (metro) $(p=0,282)$. Se clasificó a los enfermos según la distancia recorrida en $\mathbf{4}$ grupos: grupo I (distancia recorrida

Correspondencia: Dr. A. González Castro. Departamento de Medicina Intensiva. Hospital Universitario Marqués de Valdecilla.

Avda. Valdecilla, s/n.

39008 Santander. Cantabria. España.

Correo electrónico: jandro120475@ hotmail.com

Manuscrito aceptado el 8-VI-2007. menor de 100 metros), grupo II (distancia recorrida entre 101-200 metros), grupo III (distancia recorrida entre 201 y 300 metros) y grupo 4 (distancia mayor de 301 metros). No se encontraron diferencias significativas al comparar las curvas de supervivencia entre los grupos $(p=0,709)$. EI análisis multivariante tampoco aportó significación estadística.

Conclusión. La distancia recorrida en la PM6M realizada en el protocolo de estudio de candidatos a trasplante pulmonar no creemos que sea útil como marcador de mortalidad a largo plazo en los enfermos sometidos a recambio pulmonar.

PALABRAS CLAVE: prueba de marcha de los 6 minutos, trasplante pulmonar, marcadores pronósticos.

\section{EVALUATION OF THE PRE-OPERATIVE EXERCISE CAPACITY AS SURVIVAL MARKER IN THE LUNG TRANSPLANT RECIPIENTS}

Objective. To make an independent evaluation of the capacity for exercise as a predictive factor in lung transplant recipients.

Design. Retrospective study.

Scope. Lung transplant unit of the Intensive Care Unit (ICU) of a university hospital.

Patients. We analyzed 132 lung transplants in a total of 130 patients. The patients were monitorized up to June 18, 2005 or their death.

Variables of interest. An evaluation was made of their pre-operative exercise capacity with the 6-minute walking test (6MWT). Long-term survival in relationship with the walking test value was also assessed. The survival curves were estimated using the Kaplan-Meier method. Comparison between curves was made with the Log-Rank meth- 
GONZÁLEZ CASTRO A ET AL. VALORACIÓN DE LA CAPACIDAD PREOPERATORIA AL EJERCICIO COMO FACTOR PREDICTIVO DE SUPERVIVENCIA EN ENFERMOS SOMETIDOS A TRASPLANTE PULMONAR

ods and the hazard ratios were estimated by Cox regression.

Results. The association between mortality and the walking test value showed a hazard ratio of 0.99 (95\% Cl: 0.99-1.00) per metric unit (meter), (p $=0.282$ ). The patients were classified according to the distance covered in the $\mathbf{4}$ groups: group 1 (distance covered less than 100 meters), group II (distance covered between 101-200 meters), group III (distance covered between 201 and 300 meters) and group IV (distance greater than 301 meters). No significant differences were found when comparing the survival curves of the four groups $(p=$ 0.709 ). The multivariant analysis also did not show any statistical significance.

Conclusions. We do not believe that the distance covered in the 6MWT conducted in the study protocol in the lung transplant candidates is useful as a marker to predict long-term mortality in patients undergoing lung transplant.

KEY WORDS: 6 minute walking test, lung transplant, prognostic markers.

\section{INTRODUCCIÓN}

Las pruebas de esfuerzo tienen múltiples indicaciones en el campo de la neumología, y una de ellas es la evaluación preoperatoria en la cirugía torácica. Uno de los objetivos de estas pruebas es conocer el grado de limitación de tolerancia al ejercicio y estimar el riesgo de muerte o complicación postoperatoria. Las pruebas de esfuerzo están incluidas en todos los protocolos de evaluación de candidatos a trasplante, pero no hay consenso sobre el método ideal para evaluar la tolerancia al ejercicio. Generalmente se utiliza de rutina la prueba de marcha de los 6 minutos (PM6M), el tapiz rodante con el protocolo de Bruce modificado o la prueba de subir escaleras ${ }^{1-6}$.

La PM6M es un ejemplo de prueba de esfuerzo que se fundamenta en la medición de la distancia recorrida en terreno llano, durante un período de tiempo preestablecido. Para poder llevar a cabo las pruebas de marcha, la Sociedad Española de Neumología y Cirugía Torácica recomienda disponer de un corredor absolutamente plano y con una longitud igual o superior a 30 metros, preferentemente no transitado, y realizarse a una temperatura agradable. La prueba se llevará a cabo recorriendo de ida y de vuelta un tra-
TABLA 1. Escala de disnea de Borg (modificada)

\begin{tabular}{|c|c|}
\hline Puntuación & Disnea observada \\
\hline 0 & Nada \\
1 & Muy leve \\
2 & Leve \\
3 & Moderada \\
4 & Algo grave \\
5 & Grave \\
6 & - \\
7 & Muy grave \\
8 & - \\
9 & Muy, muy grave \\
10 & Máxima \\
\hline
\end{tabular}

mo de corredor de $30 \mathrm{~m}$ de longitud, que estará delimitado por señales de tipo conos de tráfico. Estas señales se colocarán a una distancia de 29 m entre sí, dejando 0,5 $\mathrm{m}$ en cada extremo para que el paciente pueda girar (fig. 1). El paciente deberá ir en compañía del examinador, quien previamente le ha informado de las características de la prueba. En condiciones basales, se tomarán los signos vitales (frecuencia cardíaca y saturación de oxígeno $\left[\mathrm{SaO}_{2}\right]$ en reposo) y se registrará el grado de disnea y de fatiga de las extremidades inferiores según escala de Borg modificada (tabla 1). Antes de comenzar la caminata se recordará al paciente la idea de recorrer la mayor distancia posible en 6 min. La prueba continúa mientras el paciente presente una $\mathrm{SaO}_{2} \geq 85 \%$ y se encuentre asintomático, siempre bajo el criterio del examinador. Una vez que el paciente se ha detenido, el examinador se acercará para registrar, lo antes posible, los datos finales de la prueba: $\mathrm{SaO}_{2}$, pulso, grado de disnea y fatiga de extremidades inferiores según la escala de Borg modificada. Se registrarán el número de recorridos completos realizados y la distancia recorrida en el último tramo hasta el punto donde se detuvo. Se contabilizará el número total de metros recorridos ${ }^{6}$.

Constituye una manera simple de valoración clínica en pacientes graves por su factibilidad, reproducibilidad, aceptación y estandarización. La marcha durante 6 minutos constituye una prueba de ejercicio submáxima, siendo considerada una prueba de carga constante ${ }^{7}$.

El protocolo preoperatorio de los enfermos candidatos a trasplante pulmonar (TP) en nuestro hospital incluye de manera rutinaria la PM6M en un intento de evaluar la capacidad funcional midiendo la distancia recorrida durante un periodo de tiempo.

El objetivo del presente estudio fue evaluar de manera independiente, como único factor, la capacidad

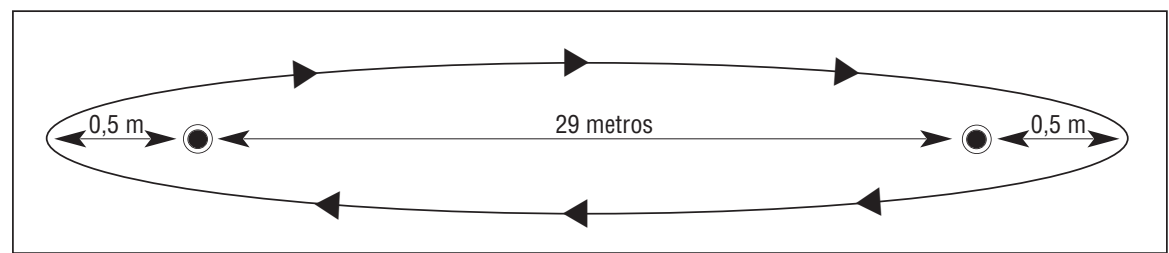

Figura 1. Recorrido a seguir durante la prueba de marcha de 6 minutos. 
al ejercicio como marcador predictivo de supervivencia en el TP.

\section{PACIENTES Y MÉTODOS}

El estudio realizado es un análisis retrospectivo de la cohorte de pacientes a los que se ha realizado un TP en el Hospital Marqués de Valdecilla entre el 29 de marzo de 1997 y el 18 de junio de 2005.

Durante el periodo definido se han realizado 132 TP en un total de 130 enfermos. El seguimiento de cada caso se realizó hasta fecha de 18 de junio de 2005 o fallecimiento del paciente.

Los hallazgos clínicos, analíticos y evolutivos de cada uno de los enfermos fueron revisados retrospectivamente a partir del historial clínico de cada uno de ellos, procedente de los archivos de historias clínicas del hospital y de los registros de la coordinación de trasplantes en lo referente a los datos de donación. Se recogieron las siguientes variables: datos de filiación (nombre con apellidos y el número de historia clínica), demográficos (edad, sexo, grupo sanguíneo y fecha de trasplante), variables clínico-funcionales del receptor (enfermedad que motivó el TP, situación de alarma cero, registro de PM6M, uso previo de corticoides y antidepresivos, enfermedad osteoporótica previa y tipo de trasplante realizado), variables de laboratorio (albúmina, prealbúmina, hemoglobina, hematocrito, protrombina, glucosa, urea, creatinina, sodio y potasio), evolutivas (transfusión de hemoderivados, presión arterial sistólica, presión arterial diastólica, relación $\mathrm{PaO}_{2} / \mathrm{FiO}_{2}$ al ingreso, a las doce y a las veinticuatro horas, presión pulmonar sistólica, presión pulmonar diastólica, edema de reperfusión, uso de inotrópicos, hemofiltración, prostaglandinas y/u óxido nítrico, régimen inmunosupresor, régimen de profilaxis antibiótica, horas de ventilación mecánica en Unidad de Cuidados Intensivos (UCI) y horas de ventilación mecánica hasta extubación, estancia en UCI, dehiscencia de suturas, reintervención por sangrado en 24 horas, exitus, fecha de exitus, causa de exitus y fecha de último seguimiento), variables en relación al donante (edad, sexo, causa de muerte cerebral, relación $\mathrm{PaO}_{2} / \mathrm{FiO}_{2}$, horas de ventilación mecánica, cultivos positivos, fumador, antigenemia citomegalovirus) y variables del acto quirúrgico (tiempo de cirugía, tiempo de isquemia de primer y segundo pulmón, cirujano, uso de circulación extracorpórea [CEC] y líquido de preservación).

La PM6M fue realizada por el Servicio de Neumología de nuestro hospital. La prueba fue efectuada siguiendo el protocolo modificado de Guyatt et $\mathrm{al}^{8,9}$.

El análisis estadístico se llevó a cabo de la siguiente manera: las medias se compararon mediante $\mathrm{t}$ de Student o ANOVA; las proporciones se compararon mediante la prueba de Chi-cuadrado o prueba de Fisher. En el análisis de supervivencia se consideró la muerte como efecto principal (end point); los pacientes que no habían fallecido en el último seguimiento fueron considerados como censurados. Las curvas de supervivencia se estimaron por el método de Kaplan-Meier; la comparación entre curvas se hi-

\begin{tabular}{|c|c|}
\hline Edad, años & $52 \pm 11$ \\
\hline Hombres & $66(69 \%)$ \\
\hline Trasplante bipulmonar & $65(68 \%)$ \\
\hline \multicolumn{2}{|l|}{ Enfermedad que motivó el trasplante } \\
\hline Enfisema & $37(39 \%)$ \\
\hline Fibrosis pulmonar & $28(29 \%)$ \\
\hline Fibrosis quística del páncreas & $2(2 \%)$ \\
\hline Retrasplante & $1(1 \%)$ \\
\hline Hipertensión pulmonar primaria & $2(2 \%)$ \\
\hline Déficit de alfa 1 antitripsina & $10(10 \%)$ \\
\hline Otros & $15(16 \%)$ \\
\hline Albúmina sérica, $\mathrm{g} / \mathrm{dl}$ & $3,6 \pm 0,7$ \\
\hline $\mathrm{PaO}_{2} / \mathrm{FiO}$, tras 24 horas de ingreso & $249 \pm 119$ \\
\hline Estancia en UCI, días & $12 \pm 17$ \\
\hline \multicolumn{2}{|l|}{ Características del donante } \\
\hline Edad, años & $39 \pm 13$ \\
\hline $\mathrm{PaO}_{2} / \mathrm{FiO}_{2}$ & $456 \pm 84$ \\
\hline Tiempo de cirugía, minutos & $367 \pm 100$ \\
\hline
\end{tabular}

Los resultados se expresan como media \pm desviación estándar.

UCI: Unidad de Cuidados Intensivos; $\mathrm{PaO}_{2} / \mathrm{FiO}_{2}$ : presión arterial de oxígeno/fracción inspiratoria de oxígeno.

zo por el método de Log-Rank y las hazard ratios se estimaron por regresión de Cox. El ajuste de factores de confusión se realizó también por regresión de Cox; los posibles factores de confusión seleccionados fueron edad del receptor (como variable continua), causa del trasplante (categórica), relación $\mathrm{PaO}_{2} / \mathrm{FiO}_{2}$ del donante (continua), tiempo de isquemia del primer pulmón (continua), albúmina sérica (continua), año del trasplante (continua), tiempo de CEC (continua) y tipo de trasplante (dicotómica: uni o bilateral).

\section{RESULTADOS}

La edad media de los receptores de TP fue de $52,35 \pm 11,95$ años, con una mediana de 55 años. La causa de trasplante fue el enfisema pulmonar en el $36,4 \%$ de los casos (48 pacientes), la fibrosis pulmonar en el 31,1\% (41 pacientes), la fibrosis quística en el 3,8\% (5 pacientes), la hipertensión pulmonar primaria en el 3,0\% (4 pacientes), el re-trasplante en el $1,5 \%$ ( 2 pacientes), el déficit de alfa 1 antitripsina en el $9,1 \%$ (12 pacientes) y en el 14,4\% (19 pacientes) el trasplante obedeció a otras causas. En 82 ocasiones se realizó un trasplante bipulmonar y en 50 el trasplante fue unipulmonar.

De los 132 trasplantes realizados, no fue posible la realización de la PM6M en 35 ocasiones, debido principalmente a contraindicaciones por la situación clínica del enfermo. Por tanto, los datos mostrados y analizados son sobre 95 pacientes. Las principales características de la muestra se observan en la tabla 2 .

El valor medio de la PM6M fue de 306,3 + 119,7 metros, con una mediana de 317 metros y unos valores de los percentiles 25-75 de 215-390 metros respectivamente.

El estudio de asociación a mortalidad de la PM6M mostró un riesgo relativo de 0,99 (intervalo de confianza [IC] del 95\%: 0,99-1,00) por cada unidad de medida métrica (metro) $(\mathrm{p}=0,282)$. 
GONZÁLEZ CASTRO A ET AL. VALORACIÓN DE LA CAPACIDAD PREOPERATORIA AL EJERCICIO COMO FACTOR PREDICTIVO DE SUPERVIVENCIA EN ENFERMOS SOMETIDOS A TRASPLANTE PULMONAR

TABLA 3. Características de los enfermos de la muestra distribuidos por grupos según la distancia recorrida en la prueba de marcha de los 6 minutos

\begin{tabular}{|c|c|c|c|c|c|}
\hline & Grupo $\mathrm{I}(<100) \mathrm{n}=4$ & Grupo II $(101-200) n=17$ & Grupo III $(201-300) n=23$ & Grupo IV $(>301) n=51$ & $\mathrm{p}$ \\
\hline Edad, años & $57 \pm 4$ & $54 \pm 7$ & $54 \pm 9$ & $49 \pm 13$ & 0,14 \\
\hline Varones & $4(100 \%)$ & $10(59 \%)$ & $17(74 \%)$ & $35(68 \%)$ & 0,41 \\
\hline Trasplante bipulmonar & $3(75 \%)$ & $11(65 \%)$ & $18(78 \%)$ & $35(68 \%)$ & 0,78 \\
\hline Enfermedad que motivó el trasplante & & & & & 0,13 \\
\hline Enfisema & $3(75 \%)$ & $10(59 \%)$ & $11(48 \%)$ & $13(25 \%)$ & \\
\hline Fibrosis pulmonar & & $3(18 \%)$ & $9(39 \%)$ & $16(31 \%)$ & \\
\hline Fibrosis quística del páncreas & & & & $2(4 \%)$ & \\
\hline Retrasplante & & $1(6 \%)$ & & $1(2 \%)$ & \\
\hline Hipertensión pulmonar primaria & & & & $1(2 \%)$ & \\
\hline Déficit de alfa 1 antitripsina & $1(25 \%)$ & $3(18 \%)$ & $2(9 \%)$ & $4(8 \%)$ & \\
\hline Otros & & & $1(4 \%)$ & $14(27 \%)$ & \\
\hline Albúmina sérica, g/dl & $3,7 \pm 0,4$ & $3,4 \pm 0,8$ & $3,6 \pm 0,5$ & $3,7 \pm 0,8$ & 0,99 \\
\hline $\mathrm{PaO}_{2} / \mathrm{FiO}_{2}$ tras 24 horas de ingreso & $356 \pm 115$ & $317 \pm 117$ & $301 \pm 127$ & $278 \pm 219$ & 0,44 \\
\hline Estancia en UCI, días & $17 \pm 18$ & $13 \pm 16$ & $16 \pm 17$ & $10 \pm 18$ & 0,53 \\
\hline Mortalidad global & $1(25 \%)$ & $7(41 \%)$ & $12(52 \%)$ & $21(41 \%)$ & 0,70 \\
\hline \multicolumn{6}{|l|}{ Características del donante } \\
\hline Edad, años & $36 \pm 17$ & $37 \pm 16$ & $37 \pm 12$ & $42 \pm 12$ & 0,31 \\
\hline $\mathrm{PaO}_{2} / \mathrm{FiO}_{2}$ & $470 \pm 76$ & $494 \pm 68$ & $452 \pm 65$ & $444 \pm 94$ & 0,19 \\
\hline Tiempo de cirugía, minutos & $388 \pm 41$ & $347 \pm 107$ & $392 \pm 107$ & $361 \pm 97$ & 0,50 \\
\hline
\end{tabular}

Valor de p: prueba ANOVA y Chi-cuadrado o prueba de Fisher.

UCI: Unidad de Cuidados Intensivos; $\mathrm{PaO}_{2} / \mathrm{FiO}_{2}$ : presión arterial de oxígeno/fracción inspiratoria de oxígeno.

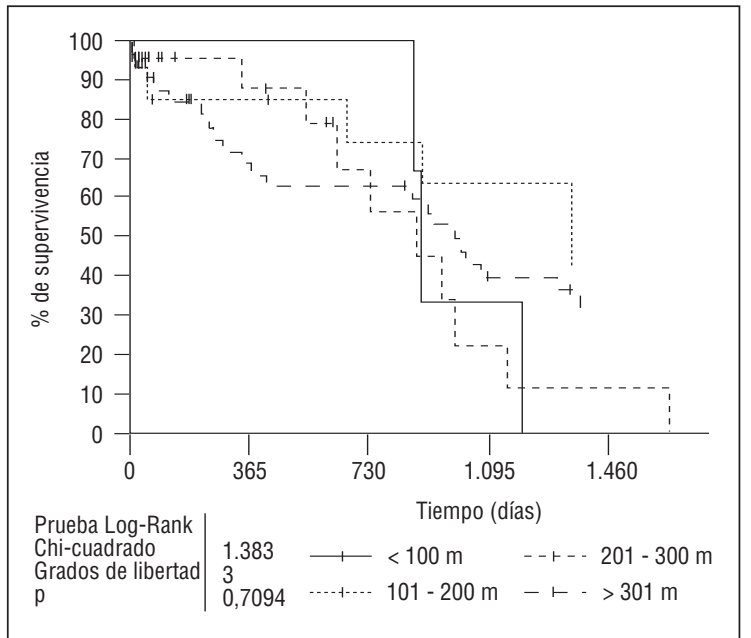

Figura 2. Curvas de supervivencia de Kaplan-Meier para los diferentes grupos de enfermos. Comparación mediante el método de Log-Rank.

Se clasificó a los enfermos según la distancia recorrida en 4 grupos: grupo I (distancia recorrida menor de 100 metros), grupo II (distancia recorrida entre 101-200 metros), grupo III (distancia recorrida entre 201 y 300 metros) y grupo 4 (distancia mayor de 301 metros). Las principales características de cada grupo se muestran en la tabla 3 .

Se realizó un análisis de supervivencia con puntos de corte anuales, en cada uno de los grupos de enfermos (fig. 2). La supervivencia al año, al segundo y al tercer año fue del 75\% (IC 95\% 33\%-100\%) para el grupo I en los tres puntos de corte. En el grupo II fueron del $62 \%$ (IC $95 \% 38 \%-86 \%$ ), 55\% (IC 95\% 30\%$80 \%$ ) y $55 \%$ (IC $95 \% 30 \%-80 \%$ ) respectivamente. En el tercer grupo se obtuvieron valores del $60 \%$ (IC 95\% $39 \%-81 \%$ ), $42 \%$ (IC $95 \%$ 20\%-64\%) y $42 \%$ (IC $95 \%$
20\%-64\%) respectivamente. Finalmente, en el grupo IV el porcentaje de supervivencia al año fue del $68 \%$ (IC 95\% 54\%-82\%), del 64\% (IC 95\% 49\%-79\%) en el segundo año y del 59\% (IC 95\% 42\%-76\%) al tercer año. No se encontraron diferencias significativas $(\mathrm{p}=0,709)$.

La asociación entre mortalidad y la distancia recorrida en la PM6M pretrasplante, tomando como referencia el valor menor de 100 metros, se muestra en la tabla 4. Como se aprecia en la tabla, no se encuentra asociación estadísticamente significativa ni en el análisis univariante ni cuando se ajustó para otras variables clínicas.

\section{DISCUSIÓN}

El objetivo de la prueba de esfuerzo en la evaluación de los enfermos candidatos a TP debiera ser el conocer el grado de limitación de tolerancia al ejercicio y la estimación del riesgo de muerte después de la cirugía $^{9-13}$. Los datos presentados en este trabajo evidencian que la limitación al ejercicio en el periodo pretrasplante pulmonar no estima el riesgo de muerte a largo plazo.

El mecanismo fisiopatológico por el que la prueba de esfuerzo se ha relacionado con la morbimortalidad implica el aumento de las demandas funcionales de los sistemas respiratorio, circulatorio y del transporte de oxígeno durante el ejercicio, que pudiera correlacionarse con las demandas funcionales en la cirugía de tórax, motivo por el cual estimaría la reserva de los sistemas implicados antes de que la reserva de $\mathrm{O}_{2}$ sea insuficiente. Sin embargo, es posible que la prueba de esfuerzo no refleje más que el estado general del paciente $^{13-16}$. Por otro lado, el principal inconveniente de esta prueba es que existe un efecto de aprendizaje a medida que se realicen sucesivamente caminatas. En este sentido, en un estudio que ha evaluado el 
GONZÁLEZ CASTRO A ET AL. VALORACIÓN DE LA CAPACIDAD PREOPERATORIA AL EJERCICIO COMO FACTOR PREDICTIVO DE SUPERVIVENCIA EN ENFERMOS SOMETIDOS A TRASPLANTE PULMONAR

TABLA 4. Asociación entre mortalidad y distancia recorrida en la prueba de marcha de los 6 minutos

\begin{tabular}{|l|c|c|c|c|}
\hline Distancia (metros) & Riesgo relativo (IC 95\%) & $\mathrm{p}$ & Riesgo relativo ajustado* (IC 95\%) & $\mathrm{p}$ \\
\hline$\leq 100$ & 1 & & 1 & 0,51 \\
$101-199$ & $0,85(0,36-1,98)$ & 0,7 & $1,86(0,29-12,0)$ & 0,58 \\
$200-299$ & $0,96(0,48-1,94)$ & 0,92 & $1,42(0,41-4,88)$ & 0,31 \\
$\geq 300$ & $0,69(0,36-1,30)$ & 0,25 & $0,57(0,19-1,70)$ & \\
\hline
\end{tabular}

*Ajustado por edad del receptor (variable continua), causa del trasplante (categórica), presión arterial de oxígeno/fracción inspiratoria de oxígeno del donante (continua), tiempo de isquemia del primer pulmón (continua), albúmina sérica (continua), año del trasplante (continua), uso de circulación extracorpórea (dicotómica) y tipo de trasplante (dicotómica: uni o bilateral). IC 95\%: intervalo de confianza al 95\%.

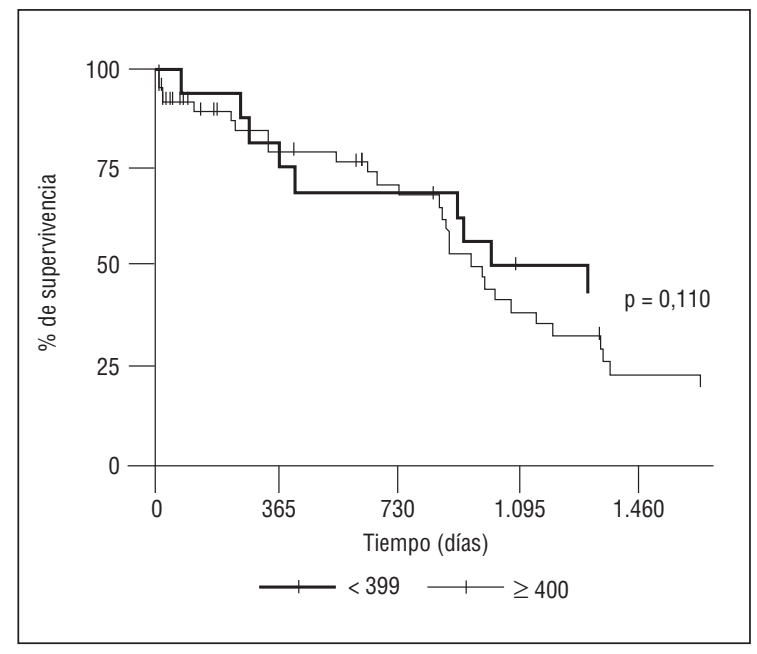

Figura 3. Curvas de Kaplan-Meier para enfermos con una distancia recorrida superior o igual a los 400 metros y enfermos con distancia menor de 399.

efecto de aprendizaje, Knox et $\mathrm{al}^{17}$ reportan que cuando se realiza la prueba en múltiples ocasiones en un tiempo corto, la distancia caminada se puede incrementar hasta un $33 \%$, solamente por el efecto de aprendizaje. En nuestra cohorte de enfermos, el conocimiento, y por tanto el aprendizaje en la prueba, se encontraba en porcentajes cercanos al $90 \%$. Este hecho podría justificar el escaso número de enfermos en nuestra cohorte que recorrieron una distancia inferior a los 100 metros. De hecho, el $72 \%$ de los pacientes de la muestra recorrieron una distancia mayor a los 200 metros. La sobreestimación de este parámetro justificaría la misma supervivencia encontrada en los diferentes grupos de pacientes.

Sin embargo, De Soyza et al demostraron la existencia de correlación entre el valor en litros de la fracción de eyección espiratoria en 1 segundo y la distancia caminada en la PM6M $(\mathrm{p}=0,0004)^{18}$. Además, la intolerancia al ejercicio es el síntoma más común de los candidatos a TP. Por tanto, la valoración de la capacidad de ejercicio debiera ser útil para evaluar la progresión de la enfermedad y el momento apropiado para el trasplante ${ }^{19,20}$.

En relación a esta afirmación, Kadikar et al, en una serie de 145 enfermos, encontraron que la PM6M presentaba una sensibilidad del $80 \%$ y una especificidad del $27 \%$ para predecir mortalidad en aquellos enfermos con valores de PM6M menores de 400 me- tros. Afirmaron que un valor de 400 metros en el estudio preoperatorio de los candidatos a trasplante representaba un umbral razonable para la inclusión definitiva de estos enfermos en la lista de trasplantes ${ }^{21}$. En nuestra serie, únicamente 22 de los 132 enfermos presentó un valor superior a 400 metros. Al realizar análisis de supervivencia clasificando a los enfermos en dos grupos (aquellos con una distancia recorrida mayor de 400, y otro grupo de distancia menor de 399) no encontramos diferencias significativas entre ambos grupos (fig. 3).

El principal estudio que valora la asociación existente entre la distancia recorrida en la PM6M preoperatoriamente y la mortalidad de enfermos sometidos a TP es el de Lederer et $\mathrm{al}^{22}$. A diferencia de nuestra serie, su trabajo únicamente incluye 454 enfermos afectos de fibrosis pulmonar idiopática en lista de espera para TP. En dicho análisis, el grupo de pacientes que recorrieron una distancia inferior a 207 metros presentó un riesgo de muerte ajustado para otras variables clínicas relevantes (edad, raza, hipertensión pulmonar, diabetes, etc.) 4,7 veces mayor (IC 95\%: 2,5 a 8,9) que los pacientes que recorrieron más de 207 metros. En nuestra serie existen 28 enfermos afectos de fibrosis pulmonar idiopática. De los 28 enfermos, cuatro presentan un valor inferior al umbral de 207 metros y 24 un valor superior. La mortalidad global en el primer subgrupo fue del $75 \%$ frente al $50 \%$ en el segundo subgrupo $(\mathrm{p}=$ $0,600)$. Por un lado la heterogeneidad de nuestra muestra, y por otro el hecho de que nuestros enfermos hayan sido sometidos a trasplante y no sean únicamente candidatos de la lista de espera, hace difícilmente comparables nuestros resultados con el estudio de Lederer et $\mathrm{al}^{22}$.

Otro hecho relevante a la hora de interpretar los resultados fue la imposibilidad de realizar la prueba en 35 enfermos principalmente por imposibilidad física y riesgo de complicación evidente. Estos enfermos no fueron incluidos en el análisis estadístico y sin duda sesgan la interpretación de los datos, ya que se supone que estos pacientes obtendrían un valor en la prueba por debajo de los 100 metros.

Existen trabajos en la literatura que demuestran una relación entre las pruebas de esfuerzo «simple» (subir escaleras y/o caminar una determinada distancia) con la aparición de complicaciones en los postoperatorios de diferentes cirugías de tórax. La ventaja de estas pruebas es que son sencillas y baratas, pero tienen el inconveniente de la escasa estandarización. No obstante, estas pruebas que evalúan la tolerancia 
al esfuerzo siguen utilizándose hoy día e influyen en la decisión de operar ${ }^{2,3,19,23-26}$.

Sin embargo, en el campo del TP no hemos encontrado ningún estudio que valorase la distancia recorrida en la PM6M realizada en el estudio de los candidatos como marcador pronóstico de mortalidad a largo plazo en los enfermos sometidos a un TP.

En resumen, la PM6M realizada en el protocolo de estudio de candidatos a trasplante no creemos que sea útil como marcador de mortalidad a largo plazo en los enfermos sometidos a recambio pulmonar. No obstante, podría ser de utilidad la individualización por patologías y características basales de estos enfermos para ajustar la utilidad de esta prueba diagnóstica. El hecho del aprendizaje en la realización de la prueba podría suponer un sesgo a la hora de interpretar los valores obtenidos.

\section{Declaración de conflicto de intereses}

Los autores han declarado no tener ningún conflicto de intereses.

\section{BIBLIOGRAFÍA}

1. Dales RE, Dionne G, Leech JA, Lunau M, Schweitzer I. Preoperative prediction of pulmonary complications following thoracic surgery. Chest. 1993;104:155-9.

2. Colman NC, Schraufnagel DE, Rivington RN, Pardy RL. Exercise testing in the evaluation of patients for lung resection. Am Rev Respir Dis. 1982;125:604-6.

3. Brunelli A, Fianchini A. Stair climbing test in lung resection candidates with low predicted postoperative FEV1. Chest. 2003;124:1179.

4. Bolliguer CT, Jordan P, Soler M, Stulz P, Tamm M, Wyser $\mathrm{C}$, et al. Pulmonary function and exercise capacity after lung resection. Eur Respir J. 1996;9:415-21.

5. Folguering $\mathrm{H}$, Palange $\mathrm{P}$, Anderson $\mathrm{S}$. Clinical exercise testing with reference to lung diseases: indications and protocols. Eur Respir Mon. 1997;6:51-71

6. Grupo de Trabajo de la SEPAR. Manual SEPAR de procedimientos. Pocedimientos de evaluación de la función pulmonar II. $1^{\mathrm{a}}$ ed. Publicaciones Permanyer; 2004.

7. Roca J, Burgos F, Casan P, Ortega F, Puente Maestu L, Togores B. Pruebas de ejercicio cardiopulmonar. Arch Bronconeumol. 2001;37:247-68.

8. Guyatt GH, Pugsley SO, Sullivan MJ, Thompson PJ, Berman LB, Jones NL, et al. Effect of encouragement on walking test performance. Thorax. 1984;39:818-22.

9. Grupo de Trabajo de la SEPAR. Cardiopulmonary exercise tests. Arch Bronconeumol. 2001;37:247-68.
10. Bisson A, Stern M, Caubarrere I. Preparation of high-risk patients for major thoracic surgery. Chest Surg Clinics. 1998;8: $541-55$.

11. Puente-Maestu L, Ruiz Martín JJ. La prueba de esfuerzo en la cirugía de resección pulmonar. Arch Bronconeumol. 2003;39: 126-32.

12. Ribas J. Aplicabilidad de las pruebas de esfuerzo en Neumología. Arch Bronconeumol. 2000;36:44-51.

13. American Thoracic Society; American College of Chest Physicians. ATS/ACCP Statement on cardiopulmonary exercise testing. Am J Respir Crit Care Med. 2003;167:211-77.

14. Beckles MA, Spiro SG; Colice GL, Rudd RM. The physiologic evaluation of patients with lung cancer being considered for resectional surgery. Chest. 2003;123:105S-14S.

15. Eugene J, Brown SE, Light RW, Milne NE, Stemmer EA. Maximun oxygen consumption: a phisiologic guide to pulmonary resection. Surg Forum. 1982;33:260-3.

16. Ninan M, Sommers E, Landreneau RJ, Weyant R, Tobias J, Luketich J, et al. Standardized exercise oximetry predicts postpneumonectomy outcome. Ann Thorac Surg. 1997;64:328-33.

17. Knox AJ, Morrison JF, Muers MF. Reproducibility of walking test results in chronic obstructive airway disease. Thorax. 1988:43:388-92.

18. De Soyza A, Pirrie S, Corris P. The 6 minute walk test's correlation with fev1 in 50 cystic fibrosis patients referred for lung transplantation. British Thoracic Society: winter meeting 2001. Abstract p131.

19. Cahalin L, Pappagianopoulos P, Prevost S, Wain J, Ginns $\mathrm{L}$. The relationship of the 6-min walk test to maximal oxygen consumption in transplant candidates with end-stage lung disease. Chest. 1995;108:452-9.

20. Vasiliadis HM, Collet JP, Poirier C. Health-related qualityof-life determinants in lung transplantation. J Heart Lung Transplant. 2006:25:226-33.

21. Kadikar A, Maurer J, Kesten S. The six-minute walk test: a guide to assessment for lung transplantation. J Heart Lung Transplant. 1997;16:313-9.

22. Lederer DJ, Arcasoy SM, Wilt JS, D'Ovidio F, Sonett JR, Kawut SM. Six-minute-walk distance predicts waiting list survival in idiopathic pulmonary fibrosis. Am J Respir Crit Care Med. 2006;174:659-64.

23. Escourrou PJ, Delaperche MF, Visseaux A. Reliability of pulse oximetry during exercise in pulmonary patients. Chest. 1990;97:635-8.

24. Puente-Maestu L, Ruiz de Oña JM, Rodríguez Hermosa JL, Santa Cruz Siminiani A, Tatay E, Cubillo JM. Predicción de la capacidad de esfuerzo tras resección pulmonar en pacientes con limitación crónica al flujo aéreo. Arch Bronconeumol. 1998;34:12732.

25. Varela G, Cordovilla R, Jiménez MF, Novoa N. Utility of standardized exercise oximetry to predict cardiopulmonary morbidity after lung resection. Eur J Cardiothorac Surg 2001;19: 351-4.

26. Ribas J, Barberá JA, Roca J, Canalís E, Mateu M, Marrades $\mathrm{RM}$, et al. ¿Qué exámenes pueden ayudar en la valoración funcional del paciente con alto riesgo para la resección pulmonar? Arch Bronconeumol. 1998;34:31-4. 